\title{
Identification and characterization of NF1 splicing mutations in Korean patients with neurofibromatosis type 1
}

\author{
Mi-Ae Jang ${ }^{1}$, Young-Eun Kim², Sun Kyung Kim ${ }^{3}$, Myoung-Keun Lee ${ }^{3}$, Jong-Won $\mathrm{Kim}^{3}$ and Chang-Seok $\mathrm{Ki}^{3}$ \\ Neurofibromatosis type I (NF1) is an autosomal dominant genetic disorder caused by NF1 mutations. Although mutations \\ affecting mRNA splicing are the most common molecular defects in NF1, few studies have analyzed genomic DNA \\ (gDNA)-mRNA correlations in Korean NF1 patients. In this study, we investigated 28 unrelated NF1 patients who showed \\ splicing alterations in reverse transcription-PCR of NF1 mRNA and identified 24 different NF1 splicing mutations, 9 of which \\ were novel. These mutations can be categorized into five groups: exon skipping resulting from mutations at authentic $5^{\prime}$ and $3^{\prime}$ \\ splice sites (type I, 46\%), cryptic exon inclusion caused by deep intronic mutations (type II, $8 \%$ ), creation of new splice sites \\ causing loss of exonic sequences (type III, $8 \%$ ), activation of cryptic splice sites due to disruption of authentic splice sites \\ (type IV, 25\%) and exonic sequence alterations causing exon skipping (type V, 13\%). In total, $42 \%$ of all splicing mutations \\ did not involve the conserved AG/GT dinucleotides of the splice sites, making it difficult to identify the correct mutation \\ sites at the gDNA level. These results add to the mutational spectrum of NF1 and further elucidate the gDNA-mRNA \\ correlations of NF1 mutations.
}

Journal of Human Genetics (2016) 61, 705-709; doi:10.1038/jhg.2016.33; published online 14 April 2016

\section{INTRODUCTION}

Neurofibromatosis type 1 (NF1; Online Mendelian Inheritance in Man (MIM) 162200) is an autosomal dominant inherited disease that primarily affects the skin and nervous system. ${ }^{1} \mathrm{NF} 1$ is one of the most common genetic disorders in humans, with a birth incidence of $\sim 1$ in 2500 to 1 in 3000 , half of which are sporadic cases. ${ }^{1,2}$ The main clinical features of NF1 are café au lait spots, Lisch nodules, inguinal and/or axillary freckling, cutaneous neurofibromas and a predisposition to develop benign and malignant nervous system tumors. ${ }^{1}$

NF1 is caused by loss-of-function mutations in a tumor suppressor gene, NF1 (neurofibromin 1; MIM 613113), located on chromosome $17 \mathrm{q} 11.2$, that consists of 60 constitutive and various alternative exons over $350 \mathrm{~kb}$ of genomic DNA (gDNA). ${ }^{1}$ Previous studies have reported over 2000 different mutations in this gene. Many of these mutations arise de novo, as there is no clear hot spot. ${ }^{3}$

The majority of NF1 mutations are predicted to produce a truncated form of the NF1 protein, and $\sim 30-50 \%$ of these mutations cause splicing alterations affecting mRNA processing. ${ }^{4,5}$ The frequency of splicing errors in NF1 is very high compared with other genetic disorders. ${ }^{2}$ In Korea, a few studies have been conducted to determine the mutational spectrum of $N F 1$, but there are few data available on splicing alterations. ${ }^{6-9}$ In the present study, we performed mutation analysis of NF1 patients at both the gDNA and mRNA levels in order to reveal gDNA-mRNA correlations.

\section{MATERIALS AND METHODS}

Study subjects

We retrospectively analyzed NF1 testing results in patients referred to a tertiary referral hospital in Korea between January 2011 and August 2015 with clinical suspicion or a differential diagnosis of NF1. We enrolled 28 unrelated NF1 patients who showed splicing alterations in reverse transcription-PCR of NF1 mRNA. This study was approved by the institutional review board of Samsung Medical Center.

\section{NF1 mutation analysis}

Genomic DNA and RNA were extracted from peripheral blood leukocytes using standard protocols. Reverse transcription-PCR and complementary DNA sequencing were performed to screen for splicing alterations and coding region mutations of NF1. NF1 complementary DNA was amplified in 24 overlapping fragments by PCR using primer sets designed by the authors (Supplementary Table S1). PCR was performed on a Thermal Cycler 9700 (Applied Biosystems, Foster City, CA, USA) as follows: denaturation at $94^{\circ} \mathrm{C}$ for $5 \mathrm{~min}, 32$ cycles at $94{ }^{\circ} \mathrm{C}$ for $30 \mathrm{~s}$, at $60^{\circ} \mathrm{C}$ for $30 \mathrm{~s}$ and at $72^{\circ} \mathrm{C}$ for $30 \mathrm{~s}$, and a final extension at $72^{\circ} \mathrm{C}$ for $1 \mathrm{~min}$. The size of the PCR products was verified by electrophoresis in $2 \%$ agarose gels. The PCR products were sequenced on an ABI 3730xl capillary

\footnotetext{
${ }^{1}$ Department of Laboratory Medicine and Genetics, Soonchunhyang University Bucheon Hospital, Soonchunhyang University College of Medicine, Bucheon, Republic of Korea; ${ }^{2}$ Green Cross Genome, Yongin, Republic of Korea and ${ }^{3}$ Department of Laboratory Medicine and Genetics, Samsung Medical Center, Sungkyunkwan University School of Medicine, Seoul, Republic of Korea

Correspondence: Dr C-S Ki, Department of Laboratory Medicine and Genetics, Samsung Medical Center, Sungkyunkwan University School of Medicine, 81 Irwon-ro, Gangnam-gu, Seoul 06351, Republic of Korea.

E-mail: changski@skku.edu

Received 17 November 2015; revised 2 March 2016; accepted 17 March 2016; published online 14 April 2016
} 
sequencer (Applied Biosystems) using the BigDye Terminator v3.1 Cycle sequencing kit (Applied Biosystems). Sequences were analyzed using Sequencher software (Gene Codes, Ann Arbor, MI, USA) and were compared with the longest isoform of the reference sequence for NF1 (NM_001042492.2). If a variant or a skipped exon was detected in complementary DNA analysis, the corresponding exonic and intronic regions of gDNA were sequenced to identify the variants that caused splicing alterations. All mutations and their putative effect at the protein level have been renamed according to the Human Genome Variation Society guidelines.

\section{Classification criteria for identified NF1 splicing mutations}

We adopted the classification system of NF1 splicing mutations proposed by Wimmer et al. ${ }^{5}$ The splicing variants were classified into five categories: type I, mutations in $5^{\prime}$ and $3^{\prime}$ splice sites resulting in exon skipping; type II, deep intronic mutations that result in inclusion of cryptic exons; type III, mutations within exons that create new splice sites and result in deletion of part of an exon; type IV, mutations inactivating the canonical $5^{\prime}$ and $3^{\prime}$ splice sites and promoting the use of cryptic exonic or intronic splice sites, leading to partial deletion of an exon or partial insertion of an intron, respectively; and type $\mathrm{V}$, exonic mutations that cause exon skipping.

\section{In silico prediction of NF1 splicing mutations}

The Human Splicing Finder (HSF) system is an online bioinformatics tool for predicting splicing signals (http://www.umd.be/HSF/). ${ }^{10}$ This software combines several different algorithms such as MaxEntScan (http://genes.mit.edu/ burgelab/maxent/Xmaxentscan_scoreseq.html), ESEfinder (http://rulai.cshl. edu/cgi-bin/tools/ESE3/esefinder.cgi?process $=$ home) and RESCUE-ESE (http://genes.mit.edu/burgelab/rescue-ese/) to identify and predict the mutation effect on splicing motifs including canonical splicing sites and auxiliary sequences known to either enhance (exonic splicing enhancers (ESEs)) or repress (exonic splicing silencers) splicing. We applied the HSF system to our set of splicing mutations to determine the accuracy of the program for predicting experimentally detected mRNA effects.

\section{RESULTS}

\section{NF1 mutations producing splicing alterations}

As shown in Table 1, a total of 24 different NF1 splicing mutations were identified in 28 unrelated patients, 9 of which have not been reported previously $(38 \%, 9 / 24)$. Most mutations were unique $(88 \%$, $21 / 24)$, although $3(13 \%, 3 / 24)$ were present in more than one patient: c. $6855 \mathrm{C}>\mathrm{A}$ (3 patients), c. $4333-2 \mathrm{~A}>\mathrm{G}$ (2 patients) and c. $1466 \mathrm{~A}>\mathrm{G}$ ( 2 patients). The majority of the splicing mutations involved the conserved AG/GT dinucleotides of the splice sites $(58 \%, 14 / 24)$. The remaining mutations were found outside the intron-exon boundaries, such as deep intronic mutations and exonic mutations (42\%, 10/24).

\section{Mutation effect on mRNA processing}

NF1 splicing mutations can be classified into five categories according to the observed effect on mRNA processing (Table 1). Several representative examples of type II--V splicing mechanisms are shown in Figure 1.

\section{Mutations in intronic sequences (types I, II and IV)}

A total of 19 splicing mutations were found in intronic sequences (79\%, 19/24). Among them, exon skipping (type I) was the most common $(58 \%, 11 / 19)$. Two deep intronic mutations (type II), which induce the insertion of a fragment of an intron that is recognized as a cryptic exon, were identified (11\%, 2/19). The use of a cryptic splice site (type IV) was observed in six cases $(32 \%, 6 / 19)$ that induced either the deletion of part of an exon $(26 \%, 5 / 19)$ or the insertion of part

Table 1 Twenty-four NF1 mutations and their effects on mRNA in 28 unrelated NF1 patients

\begin{tabular}{|c|c|c|c|c|c|c|}
\hline Class & Location & DNA mutation & Effect on $m R N A$ & Predicted protein & Reference & Frequency $^{\mathrm{a}}$ \\
\hline \multirow[t]{11}{*}{ Type $1(N=11)$} & IVS6 & c. $655-2 A>G$ & r.655_730del76 & p.Ala219Asnfs*37 & Disease database $e^{b}$ & 1 \\
\hline & IVS6 & c. $655-1 G>A$ & r.655_730del76 & p.Ala219Asnfs*37 & Horn et al. ${ }^{16}$ & 1 \\
\hline & IVS9 & c. $1062+1 G>A$ & r.889_1062del174 & p.Lys297_Lys354del & Eisenbarth et al. ${ }^{17}$ & 1 \\
\hline & IVS13 & c. $1527+1 G>T$ & r.1393_1527del135 & p.Ser465_Cys509del & Pros et al. ${ }^{13}$ & 1 \\
\hline & IVS13 & c. $1527+2 \mathrm{~T}>\mathrm{C}$ & r.1393_1527del135 & p.Ser465_Cys509del & Disease database ${ }^{b}$ & 1 \\
\hline & IVS14 & c. $1642-2 \mathrm{~A}>\mathrm{T}$ & r.1642_1721del80 & p.Ala548Leufs*13 & This study & 1 \\
\hline & IVS21 & c.2851-6_2851-3del & r.2851_2990del140 & p.Leu952Cysfs*22 & Messiaen et al. ${ }^{2}$ & 1 \\
\hline & IVS21 & c. $2851-1 G>A$ & r.2851_2990del140 & p.Leu952Cysfs*22 & Sabbagh et al. ${ }^{18}$ & 1 \\
\hline & IVS23 & c. $3113+1 G>A$ & r.2991_3113del123 & p.Tyr998_Arg1038del & Purandare et al. ${ }^{19}$ & 1 \\
\hline & IVS32 & c. $4333-2 \mathrm{~A}>\mathrm{G}$ & r.4333_4430del98 & p.lle1445Valfs*4 & Raygada et al. ${ }^{20}$ & 2 \\
\hline & IVS46 & c. $6921+4 A>G$ & r.6820_6921del102 & p.Ala2274_Lys2307del & This study & 1 \\
\hline \multirow[t]{2}{*}{ Type $\|(N=2)$} & IVS12 & c. $1392+754 \mathrm{~T}>\mathrm{G}$ & r.1392_1393ins1392+755_1392+824 & Premature stop codon ${ }^{c}$ & This study & 1 \\
\hline & IVS38 & c. $5610-456 \mathrm{G}>\mathrm{T}$ & r.5609_5610ins5610-538_5610-458 & Premature stop codon ${ }^{c}$ & This study & 1 \\
\hline \multirow[t]{2}{*}{ Type III (N=2) } & E13 & c. $1466 \mathrm{~A}>\mathrm{G}$ & r.1466_1527del62 & p.Tyr489* & Wimmer et al. ${ }^{5}$ & 2 \\
\hline & E16 & c. $1748 \mathrm{~A}>\mathrm{G}$ & r.1722_1748del27 & p.Ser574_Lys583delinsArg & Valero et al. ${ }^{21}$ & 1 \\
\hline \multirow[t]{6}{*}{ Type IV $(N=6)$} & IVS2 & c. $204+1 G>A$ & r.100_204del105 & p.Val34_Met68del & Ars et al..$^{22}$ & 1 \\
\hline & IVS2 & c.204+1_204+4del & r.100_204del105 & p.Val34_Met68del & This study & 1 \\
\hline & IVS14 & c. $1642-10 A>G$ & r.1641_1642ins1642-9_1642-1 & p.Glu547_Ala548insLyslleGln & This study & 1 \\
\hline & IVS21 & c. $2850+1 G>C$ & r.2618_2850del233 & p.Lys874Phefs*4 & This study & 1 \\
\hline & IVS36 & c. $4835+1 G>A$ & r.4767_4835del69 & p.Leu1590_Arg1612del & Wimmer et al. ${ }^{5}$ & 1 \\
\hline & IVS36 & c. $4836-1 \mathrm{G}>\mathrm{A}$ & r.4836delg & p.Arg1612Serfs*12 & This study & 1 \\
\hline \multirow[t]{3}{*}{ Type V $(N=3)$} & E46 & c. $6855 \mathrm{C}>\mathrm{A}$ & r.6820_6921del102 & p.Ala2274_Lys2307del & Robinson et al. ${ }^{11}, \mathrm{~d}$ & 3 \\
\hline & E46 & c. $6904 \mathrm{C}>\mathrm{T}$ & r.6820_6921del102 & p.Ala2274_Lys2307del & Upadhyaya et al. ${ }^{12, \mathrm{~d}}$ & 1 \\
\hline & E46 & c. $6921 \mathrm{G}>\mathrm{A}\left(5^{\prime} \mathrm{ss}-1\right)$ & r.6820_6921del102 & p.Ala2274_Lys2307del & This study & 1 \\
\hline
\end{tabular}

Abbreviations: E, exon; IVS, intervening sequence; NF1, neurofibromatosis type I; ss, splice site

NF1 reference accession number: NM_001042492.2 (NP_001035957.1).

a Number of patients.

bLeiden Open Variation Database https://grenada.lumc.nI/LOVD2/mendelian_genes/home.php?select_db = NF1

${ }^{\mathrm{c}}$ The insertion of an intronic sequence leads to inclusion of an in-frame premature stop codon.

dPreviously reported as a nonsense mutation. 
of an intron $(5 \%, 1 / 19)$. Except for two deep intronic mutations (type II), 53\% of mutations were found at the $5^{\prime}$ splice site $(9 / 17)$, whereas $47 \%$ were found at the $3^{\prime}$ splice site (8/17). The most common mutated nucleotides at the $5^{\prime}$ and $3^{\prime}$ splice sites were, in decreasing order, $+1(35 \%, 6 / 17),-1(18 \%, 3 / 17)$ and -2 $(18 \%, 3 / 17)$.

\section{Mutations in exonic sequences (types III and V)}

Five different mutations were identified in the coding region that affected mRNA splicing (type III, $n=2$; type V, $n=3$ ). Two of these exonic mutations were previously classified as nonsense mutations (c.6855C > A and c.6904C $>\mathrm{T}$ in type V), ${ }^{11,12}$ but actually altered the splicing of the exon itself.

In the two different type III mutations, the nucleotide change in the exonic region induced a new $5^{\prime}$ splice site $(\mathrm{c} .1466 \mathrm{~A}>\mathrm{G})$ or $3^{\prime}$ splice site $(\mathrm{c} .1748 \mathrm{~A}>\mathrm{G})$ that led to partial deletion of the exon. All type $\mathrm{V}$ mutations were identified in exon 46; among them, one mutation occurred in the last conserved $\mathrm{G}$ sequence of exon 46 (position - 1 of the donor site), causing exon skipping (c.6921G $>$ A). Of the two remaining mutations, c. $6855 \mathrm{C}>\mathrm{A}$ and $\mathrm{c} .6904 \mathrm{C}>\mathrm{T}$, neither altered the existing normal splice site nor created a novel site. The mutations may exert their effects through altered interactions between an ESE and mRNA splicing factor.

\section{In silico prediction of NF1 splicing mutations}

In silico prediction of NF1 splicing mutations by HSF and MaxEntScan programs are summarized in Table 2. We calculated the consensus values $(\mathrm{CVs})$ of the splice sites involved in splicing alterations. In all cases where the mutations cause skipping of NF1 exons, the CVs of the mutated splice sites were lower than those of the wild-type splice site sequences. The average reduction between wild- and mutant-type CV was 35 and 127\% according to HSF and MaxEntScan, respectively. For all type II and III mutations where a novel splice site was created because of the mutation, the CV scores of the new acceptor or donor

a
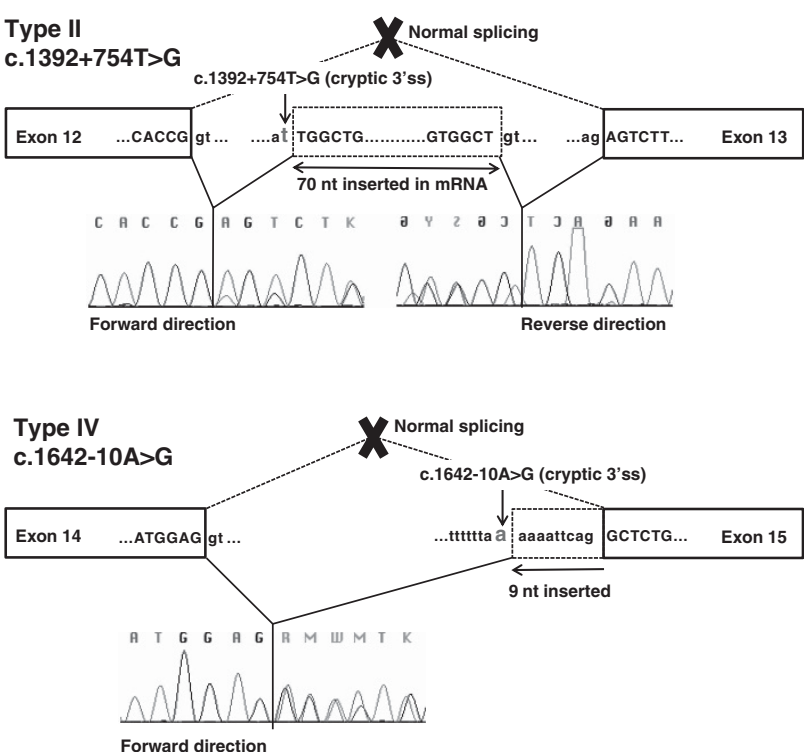

site were higher than those for the wild-type splice site. In the cases with type IV mutations, almost all mutations revealed a significant decrease in the strength of the splice site compared with the wild-type sequence. When the HSF and MaxEntScan systems were used to score the strength of the cryptic splice sites, high scores for a potential splice site were obtained for only cryptic $3^{\prime}$ splice sites (c.1642-10A $>\mathrm{G}$ and c.4836-1G>A), and not for cryptic $5^{\prime}$ splice sites. Two type V mutations, c.6855C $>\mathrm{A}$ and c.6904C $>\mathrm{T}$, were predicted by ESEfinder to decrease the score of an ESE element (Table 3). The RESCUE-ESE program predicted the creation of a novel ESE hexamer for the c. $6855 \mathrm{C}>\mathrm{A}$ mutation, but no changes for the c.6904C $>\mathrm{T}$ mutation.

\section{DISCUSSION}

Mutation analysis of NF1 for diagnostic purposes is a difficult task owing to the large size of the gene, the presence of homologous pseudogenes and the lack of clear mutational hot spots. ${ }^{2}$ Occurrence of unusual splice mutations outside of the conserved splice sites also makes the gene test challenging. ${ }^{2,4,13}$ In the present study, we observed that unusual splicing mutations are common, being present in $42 \%$ of the mutations (10/24) including intronic mutations outside the conserved AG/GT splice sites $(21 \%, 5 / 24)$ and exonic mutations $(21 \%, 5 / 24)$. Most of these unusual splicing mutations create novel splice sites or activate cryptic splice sites within exonic and intronic sequences. The proportion of unusual splicing mutations in our study was $\sim 20 \%$ higher than those of published studies. ${ }^{13}$ Therefore, our results emphasize the importance of analyzing NF1 at the mRNA level so as to clarify the primary effect of the mutation on mRNA processing. The mRNA-based assay could also be helpful in screening the complete NF1 coding sequence and avoiding nonprocessed pseudogene amplification. It has been reported that whereas $50 \%$ of the mutations at the DNA level were predicted to disrupt splicing, this proportion increased significantly to $95 \%$ when the effect of the mutation was studied at the mRNA level. ${ }^{2}$

b

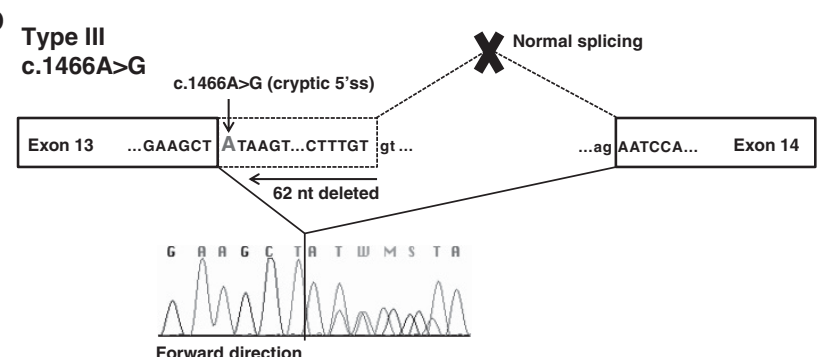

d

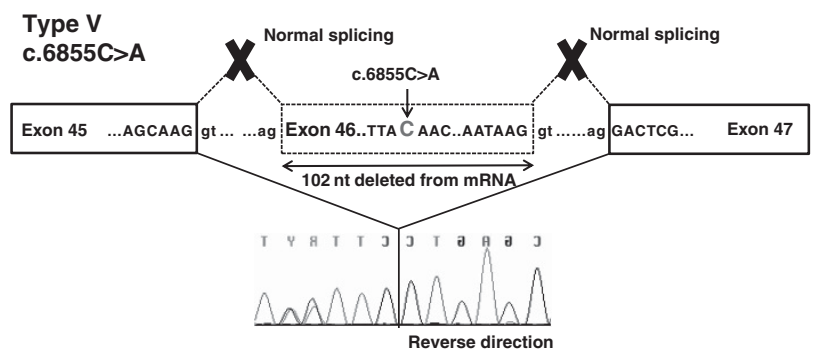

Figure 1 Schematic representations of the type II-V splicing mechanisms involved in some neurofibromatosis type I (NF1) mutations. (a) Deep intronic mutation, c. 1392+754T > G, activates a cryptic $3^{\prime}$ splice site (ss) in intron 12 that results in the introduction of a supplementary exon 70 nucleotides (nt) in length in the mRNA between exons 12 and 13. (b) Exonic mutation, c.1466A>G, creates a new 5' ss within exon 13 that gives rise to a deletion of $62 \mathrm{nt}$ from the mRNA. (c) Intronic mutation, c.1642-10A > G of intron 14, creates a new $3^{\prime}$ ss that leads to a 9-nt insertion at exon 15. (d) Exonic mutation, c.6855C $>$ A, disrupts an exonic splicing enhancer element that results in skipping of exon 46. Uppercase letters indicate exonic sequences, and lowercase letters indicate intronic sequences. A full color version of this figure is available at the Journal of Human Genetics journal online. 
Table 2 In silico analysis of NF1 splicing mutations by HSF and MaxEntScan prediction programs

\begin{tabular}{|c|c|c|c|c|c|c|c|c|}
\hline \multirow[b]{2}{*}{ Class } & \multirow[b]{2}{*}{ Mutation } & \multirow[b]{2}{*}{ Effect on $m R N A$} & \multicolumn{3}{|c|}{ Predicted scores by HSF } & \multicolumn{3}{|c|}{ Predicted scores by MaxEntScan } \\
\hline & & & WT CV & Mut CV & Variation, \% & WT score & Mut score & Variation, \% \\
\hline । & c. $655-2 A>G$ & Inact 3' ss, skip E7 & 85.99 & 57.05 & -33.66 & 8.27 & 0.32 & -96.13 \\
\hline I & c. $655-1 G>A$ & Inact 3' ss, skip E7 & 85.99 & 57.05 & -33.66 & 8.27 & -0.47 & -105.68 \\
\hline 1 & c. $1062+1 \mathrm{G}>\mathrm{A}$ & Inact 5' ss, skip E9 & 84.7 & 57.86 & -31.69 & 8.92 & 0.74 & -91.7 \\
\hline I & c. $1527+1 G>T$ & Inact 5' ss, skip E13 & 82.29 & 55.46 & -32.6 & 7.65 & -0.85 & -111.11 \\
\hline 1 & c. $1527+2 T>C$ & Inact 5' ss, skip E13 & 82.29 & 55.46 & -32.6 & 7.65 & -0.1 & -101.31 \\
\hline I & c. $1642-2 \mathrm{~A}>\mathrm{T}$ & Inact 3' ss, skip E15 & 79.57 & 50.63 & -36.37 & 4.83 & -3.53 & -173.08 \\
\hline 1 & c. $2851-6 \_2851-3 \mathrm{del}$ & Inact $3^{\prime}$ ss, skip E22 & 89.92 & 19.95 & -77.81 & 11.24 & -17.93 & -259.52 \\
\hline 1 & c. $2851-1 G>A$ & Inact $3^{\prime}$ ss, skip E22 & 89.92 & 60.98 & -32.18 & 11.24 & 2.49 & -77.85 \\
\hline I & c. $3113+1 G>A$ & Inact 5' ss, skip E23 & 96.86 & 70.02 & -27.71 & 8.83 & 0.65 & -92.64 \\
\hline 1 & c. $4333-2 A>G$ & Inact 3 ' ss, skip E33 & 80.83 & 51.89 & -35.8 & 7.92 & -0.02 & -100.25 \\
\hline 1 & c. $6921+4 A>G$ & Inact 5' ss, skip E46 & 86.73 & 78.39 & -9.62 & 8.83 & 6.44 & -27.07 \\
\hline II & c. $1392+754 \mathrm{~T}>\mathrm{G}$ & $\begin{array}{l}\text { New } 3^{\prime} \text { ss, inclusion cryptic exon of } \\
70 \text { nt between } \mathrm{E} 12 \text { and } \mathrm{E} 13\end{array}$ & 49.64 & 78.59 & +58.32 & -2.31 & 6.28 & +371.86 \\
\hline 11 & c. $5610-456 G>T$ & $\begin{array}{l}\text { New } 5^{\prime} \text { ss, inclusion cryptic exon of } \\
81 \text { nt between E38 and E39 }\end{array}$ & 70.28 & 97.11 & +38.18 & 2.87 & 10.51 & +266.2 \\
\hline III & c. $1466 A>G$ & New $5^{\prime}$ ss, deletion last 62 nt E13 & 55.79 & 82.62 & +48.09 & 0.38 & 8.56 & +2152.63 \\
\hline III & c. $1748 \mathrm{~A}>\mathrm{G}$ & New $3^{\prime}$ ss, deletion first 27 nt E16 & 62.26 & 91.2 & +46.48 & 1.06 & 9.81 & +825.47 \\
\hline IV & c. $204+1 G>A$ & $\begin{array}{l}\text { Inact } 5 \text { ' ss, use cryptic 5' ss, } \\
\text { deletion last } 105 \text { nt E2 }\end{array}$ & 93.14 & 66.3 & -28.82 & 10.113 & 1.94 & -80.85 \\
\hline IV & c.204+1_204+4del & $\begin{array}{l}\text { Inact } 5^{\prime} \text { ss, use cryptic 5' ss, } \\
\text { deletion last } 105 \text { nt E2 }\end{array}$ & 93.14 & 73.17 & -21.44 & 10.13 & 3.85 & -61.99 \\
\hline IV & c. $1642-10 A>G$ & $\begin{array}{l}\text { Inact } 3 \text { ' ss, use cryptic } 3^{\prime} \text { ss, } \\
\text { insertion } 9 \text { nt IVS14 }\end{array}$ & $\begin{array}{l}79.57 \\
(54.1)\end{array}$ & $\begin{array}{c}79.69 \\
(83.04)\end{array}$ & $+0.15(+53.49)^{a}$ & $4.83(3.58)$ & $\begin{array}{c}-2.2 \\
(12.33)\end{array}$ & $\begin{array}{c}-145.55 \\
(+244.41)^{a}\end{array}$ \\
\hline IV & c. $2850+1 G>C$ & $\begin{array}{l}\text { Inact 5' ss, use cryptic 5' ss, } \\
\text { deletion last } 233 \text { nt E21 }\end{array}$ & 85.9 & 59.07 & -31.23 & 9.65 & 1.38 & -85.7 \\
\hline IV & c. $4835+1 G>A$ & $\begin{array}{l}\text { Inact 5' ss, use cryptic 5' ss, } \\
\text { deletion last } 69 \text { nt E36 }\end{array}$ & 95.9 & 69.07 & -27.98 & 10.06 & 1.88 & -81.31 \\
\hline IV & c. $4836-1 G>A$ & $\begin{array}{l}\text { Inact } 3 \text { ' ss, use cryptic 3' ss, } \\
\text { deletion first } 1 \text { nt E37 }\end{array}$ & $\begin{array}{c}84.57 \\
(52.75)\end{array}$ & $\begin{array}{c}55.62 \\
(81.69)\end{array}$ & $\begin{array}{c}-34.23 \\
(+54.86)^{\mathrm{a}}\end{array}$ & $\begin{array}{c}11.72 \\
(-1.98)\end{array}$ & $2.97(5.97)$ & $\begin{array}{c}-74.66 \\
(+401.52)^{a}\end{array}$ \\
\hline V & c. $6921 \mathrm{G}>\mathrm{A}\left(5^{\prime} \mathrm{ss}-1\right)$ & Inact 5' ss, skip E46 & 79.51 & 50.56 & -36.41 & 3.04 & -5.7 & -287.5 \\
\hline
\end{tabular}

Abbreviations: CV, consensus value; E, exon; HSF, Human Splicing Finder; Inact, inactivation; Mut, mutant type; nt, nucleotide; ss, splice site; WT, wild type.

NF1 reference accession number: NM_001042492.2 (NP_001035957.1).

aThe consensus value of cryptic splice sites predicted by prediction programs.

Table 3 Two NF1 mutations disrupting exonic splicing enhancer elements and their in silico analysis

\begin{tabular}{|c|c|c|c|c|c|c|c|}
\hline \multirow[b]{2}{*}{ Class } & \multirow[b]{2}{*}{ Mutation } & \multirow[b]{2}{*}{ Effect on $m R N A$} & \multicolumn{4}{|c|}{ ESEfinder prediction } & \multirow[b]{2}{*}{ RESCUE-ESE prediction } \\
\hline & & & WT motif & Mut motif & Variation, \% & $S R$ protein & \\
\hline V & c. $6855 \mathrm{C}>\mathrm{A}$ & ESE site broken, skip E46 & 89.64 & 80.24 & -10.49 & SRp40 & New hexamer \\
\hline V & c. $6904 \mathrm{C}>\mathrm{T}$ & ESE site broken, skip E46 & $\begin{array}{l}93.05 \\
83.08\end{array}$ & $\begin{array}{c}78.80 \\
\text { Site broken }\end{array}$ & $\begin{array}{c}-15.32 \\
-100\end{array}$ & $\begin{array}{l}\text { SRp40 } \\
\text { SRp55 }\end{array}$ & No change \\
\hline
\end{tabular}

Abbreviations: E, exon; ESE, exon splicing enhancer; Mut, mutant type; WT, wild type.

NF1 reference accession number: NM_001042492.2 (NP_001035957.1).

The scores of individual SR protein putative recognition sites calculated by ESEfinder and the presence or absence of hexamer sequences predicted by RESCUE-ESE to function as ESE elements are provided for wild-type and mutant sequences.

In this study, we report 24 different splicing mutations of NF1 in 28 unrelated subjects suspected of having NF1. The mutations are scattered along the gene, with some exons having a higher number of mutations. It is of note that 4 splicing mutations of NF1 were clustered in the exonic and intronic boundaries of exon 46, which is located in the carboxyl terminal domain of the NF1 protein, and were observed in 6 of 28 patients $(21 \%)$. The importance of the carboxyl terminal domain and its potential role in tumor suppression are unclear; thus, the functional effect of this domain remains to be elucidated. ${ }^{14}$
In order to determine the putative mRNA effect of a given mutation, multiple software programs have been developed. In general, splice site prediction tools have higher sensitivity $(\sim 90-100 \%)$ than specificity $(\sim 60-80 \%)$ for predicting splice site abnormalities. ${ }^{15}$ To evaluate the efficiency of the prediction software for splicing mutations, we analyzed a set of 24 splicing mutations identified in our study cohort. HSF and MaxEntScan programs were able to correctly predict the disruption of the natural splice sites, and we confirmed that the mutation of the last nucleotide of an exon has a strong effect on the $5^{\prime}$ splice site, with $36.41 \% \mathrm{CV}$ reduction. 
Furthermore, exonic mutations distant from the $5^{\prime}$ and $3^{\prime}$ splice sites can activate a cryptic splice site, leading to partial exonic deletion. ESE prediction programs indicate disruption of putative ESE elements for two NF1 mutations, c.6855C $>$ A and c.6904C $>$ T. Thus, integrated analyses using bioinformatics tools were further applied to provide insight into the mechanisms of these splicing mutations caused by exonic variants, as well as other intronic variants at non-consensus splice sites.

This study has the following limitations. First, the lack of clinical information for most of the patients referred to our laboratory makes it impossible to provide genotype-phenotype correlations. In addition, large NF1 deletion mutations detected only by fluorescence in situ hybridization or multiple ligation probe amplification were not considered in our study; however, they represented only $5 \%$ of the total NF1 mutations.

Taken together, the results from our work show that the molecular basis of NF1 splicing mutations is diverse. Therefore, molecular characterization at both the gDNA and mRNA levels allowed for a better understanding of gDNA-mRNA correlations of NF1 mutations.

\section{CONFLICT OF INTEREST}

The authors declare no conflict of interest.

\section{ACKNOWLEDGEMENTS}

This study was supported by the Basic Science Research Program through the National Research Foundation of Korea (NRF) funded by the Ministry of Education, Science and Technology (2013R1A1A2009756).

1 Ferner, R. E. Neurofibromatosis 1 and neurofibromatosis 2: a twenty first century perspective. Lancet Neurol. 6, 340-351 (2007).

2 Messiaen, L. M., Callens, T., Mortier, G., Beysen, D., Vandenbroucke, I., Van Roy, N. et al. Exhaustive mutation analysis of the NF1 gene allows identification of $95 \%$ of mutations and reveals a high frequency of unusual splicing defects. Hum. Mutat. 15, 541-555 (2000)

3 Xu, W. Yang, X., Hu, X. \& Li, S. Fifty-four novel mutations in the NF1 gene and integrated analyses of the mutations that modulate splicing. Int. J. Mol. Med. 34, 53-60 (2014).

4 Ars, E., Serra, E., Garcia, J., Kruyer, H., Gaona, A., Lazaro, C. et al. Mutations affecting mRNA splicing are the most common molecular defects in patients with neurofibromatosis type 1. Hum. Mol. Genet. 9, 237-247 (2000).
5 Wimmer, K., Roca, X., Beiglbock, H., Callens, T., Etzler, J., Rao, A. R. et al. Extensive in silico analysis of NF1 splicing defects uncovers determinants for splicing outcome upon 5' splice-site disruption. Hum. Mutat. 28, 599-612 (2007).

6 Jeong, S. Y., Park, S. J. \& Kim, H. J. The spectrum of NF1 mutations in Korean patients with neurofibromatosis type 1. J. Korean Med. Sci. 21, 107-112 (2006).

7 Kim, M. J. \& Cheon, C. K. Neurofibromatosis type 1: a single center's experience in Korea. Korean J. Pediatr. 57, 410-415 (2014).

8 Ko, J. M., Sohn, Y. B., Jeong, S. Y., Kim, H. J. \& Messiaen, L. M. Mutation spectrum of NF1 and clinical characteristics in 78 Korean patients with neurofibromatosis type 1 . Pediatr. Neurol. 48, 447-453 (2013).

9 Park, S., Sohn, Y. B., Chung, I. S., Hong, J. H., Jung, E. J., Jeong, S. Y. et al. A Korean case of neurofibromatosis type 1 with an exonic splicing enhancer site mutation. J. Genet. Med. 11, 40-42 (2014).

10 Desmet, F. O., Hamroun, D., Lalande, M., Collod-Beroud, G., Claustres, M. \& Beroud, C. Human Splicing Finder: an online bioinformatics tool to predict splicing signals. Nucleic Acids Res. 37, e67 (2009).

11 Robinson, P. N., Boddrich, A., Peters, H., Tinschert, S., Buske, A., Kaufmann, D. et al. Two recurrent nonsense mutations and a $4 \mathrm{bp}$ deletion in a quasi-symmetric element in exon 37 of the NF1 gene. Hum. Genet. 96, 95-98 (1995).

12 Upadhyaya, M., Osborn, M., Maynard, J. \& Harper, P. Characterization of six mutation in exon 37 of neurofibromatosis type 1 gene. Am. J. Med. Genet 67, 421-423 (1996).

13 Pros, E., Gomez, C., Martin, T., Fabregas, P., Serra, E. \& Lazaro, C. Nature and mRNA effect of 282 different NF1 point mutations: focus on splicing alterations. Hum. Mutat. 29, E173-E193 (2008).

14 Ratner, N. \& Miller, S. J. A RASopathy gene commonly mutated in cancer: the neurofibromatosis type 1 tumour suppressor. Nat. Rev. Cancer 15 , 290-301 (2015).

15 Richards, S., Aziz, N., Bale, S., Bick, D., Das, S., Gastier-Foster, J. et al. Standards and guidelines for the interpretation of sequence variants: a joint consensus recommendation of the American College of Medical Genetics and Genomics and the Association for Molecular Pathology. Genet. Med. 17, 405-424 (2015).

16 Horn, D., Robinson, P. N., Boddrich, A., Buske, A., Tinschert, S. \& Nurnberg, P. Three novel mutations of the NF1 gene detected by temperature gradient gel electrophoresis of exons 5 and 8. Electrophoresis 17, 1559-1563 (1996).

17 Eisenbarth, I., Beyer, K., Krone, W. \& Assum, G. Toward a survey of somatic mutation of the NF1 gene in benign neurofibromas of patients with neurofibromatosis type 1. Am. J. Hum. Genet. 66, 393-401 (2000).

18 Sabbagh, A., Pasmant, E., Imbard, A., Luscan, A., Soares, M., Blanche, H. et al. NF1 molecular characterization and neurofibromatosis type I genotype-phenotype correlation: the French experience. Hum. Mutat. 34, 1510-1518 (2013).

19 Purandare, S. M., Lanyon, W. G., Arngrimsson, R. \& Connor, J. M. Characterisation of a novel splice donor mutation affecting position +1 in intron 18 of the NF-1 gene. Hum. Mol. Genet. 4, 767-768 (1995).

20 Raygada, M., Arthur, D. C., Wayne, A. S., Rennert, O. M., Toretsky, J. A. \& Stratakis, C. A. Juvenile xanthogranuloma in a child with previously unsuspected neurofibromatosis type 1 and juvenile myelomonocytic leukemia. Pediatr. Blood Cancer 54, 173-175 (2010).

21 Valero, M. C., Martin, Y., Hernandez-Imaz, E., Marina Hernandez, A., Melean, G. Valero, A. M. et al. A highly sensitive genetic protocol to detect NF1 mutations. J. Mol. Diag. 13, 113-122 (2011).

22 Ars, E., Kruyer, H., Morell, M., Pros, E., Serra, E., Ravella, A et al. Recurrent mutations in the NF1 gene are common among neurofibromatosis type 1 patients. J. Med. Genet. 40, e82 (2003).

Supplementary Information accompanies the paper on Journal of Human Genetics website (http://www.nature.com/jhg) 